Check for updates

Cite this: RSC Adv., 2018, 8, 5233

Received 15th November 2017 Accepted 24th January 2018

DOI: 10.1039/c7ra12472d

rsc.li/rsc-advances

\section{Correlating supercritical fluid extraction parameters with volatile compounds from Finnish wild mushrooms (Craterellus tubaeformis) and yield prediction by partial least squares regression analysis $\uparrow$}

\author{
Xiao Chen, ${ }^{\text {ab }}$ Nghia Huynh, ${ }^{\mathrm{b}}$ Heping Cui, ${ }^{\mathrm{a}}$ Peng Zhou, ${ }^{\mathrm{a}}$ Xiaoming Zhang (DD *a \\ and Baoru Yang ${ }^{\star b}$
}

Supercritical fluid was applied to extract volatile compounds from Finnish wild mushrooms (Craterellus tubaeformis). The effects of extraction pressure, temperature and supercritical carbon dioxide volume on extraction yield and the content of mushroom alcohols in the extracts were investigated in the range from 80 to 95 bar, 35 to $55^{\circ} \mathrm{C}$ and 30 to $70 \mathrm{~mL}$, respectively. The correlation between extracted volatile compounds and supercritical fluid extraction parameters was studied and prediction models of ten extracted aroma compounds were established by partial least squares regression (PLSR). The calibrated and validated models of 2 -octen-1-ol $\left(R_{-}\right.$cal $=0.96, R_{-}$cal ${ }^{2}=0.91, R_{-}$val $=0.94, R_{-}$val $\left.{ }^{2}=0.88\right)$ and geranyl acetone $\left(R_{- \text {cal }}=0.96, R_{\text {ccal }}{ }^{2}=0.92, R_{\text {_val }}=0.95, R_{\text {_val }}{ }^{2}=0.90\right)$ were satisfactory, and had the predictive capability of $88 \%$ and $92 \%$, respectively. Moreover, the predictive equations for other extracted aroma compounds were also proved to be sufficiently accurate. Hence, the present study provides useful reference for extraction of volatile compounds from mushrooms using supercritical fluid for further industrial applications.

\section{Introduction}

The mushroom market has grown exponentially in the last few years ${ }^{1}$ since mushrooms have been used as food and/or nutraceuticals. ${ }^{2}$ Europe has accounted for the largest mushroom market, which has been estimated to reach the highest compound annual growth rate (CAGR) of $10.8 \%$ between 2014 and 2019. ${ }^{1}$ Finland has rich sources of wild edible mushrooms and consumption is increasing due to the exotic and clean natural growth environment. ${ }^{3}$ Craterellus tubaeformis ${ }^{4}$ also known as Cantharellus tubaeformis, is an edible fungus growing widely in Finland. Previous studies have shown that it contains high amounts of ergocalciferol compared with some cultivated mushrooms and can be recommended as a natural source of vitamin D for humans. ${ }^{5,6}$ Despite the high content of bioactive compounds and the great potential in the international market, the utilization of this Finnish wild mushroom is still limited.

${ }^{a}$ State Key Laboratory of Food Science and Technology, School of Food Science and Technology, Jiangnan University, Lihu Road 1800, Wuxi, Jiangsu 214122, China. E-mail: xmzhang@jiangnan.edu.cn; Tel: +86-510-85919106

${ }^{b}$ Food Chemistry and Food Development, Department of Biochemistry, University of Turku, FIN-20014 Turku, Finland. E-mail: baoru.yang@utu.fi; Tel: +358 23336844

$\dagger$ Electronic supplementary information (ESI) available. See DOI: $10.1039 / \mathrm{c} 7 \mathrm{ra} 12472 \mathrm{~d}$
For many decades mushrooms have been widely utilized as popular ingredients in various cuisines around the world because of their unique aroma and taste ${ }^{7}$ volatile compounds are the major contributor to the characteristic mushroom flavour. ${ }^{8}$ Hundreds of volatile compounds have been identified in edible mushrooms. The most important volatiles are mushroom alcohols, including 1-octen-3-ol, 2-octen-1-ol, 3-octanol, and 1-octanol, which have been identified as the main compounds responsible for the unique aroma and flavour. ${ }^{9}$ In particular, 1-octen-3-ol (unsaturated alcohol) has been found in almost all mushroom species. It is formed during the enzymatic breakdown of linoleic acid, ${ }^{\mathbf{1 0 , 1 1}}$ and has been considered as the main component responsible for the characteristic flavour of most edible mushrooms. ${ }^{12-15}$ The mushroom aroma is much wanted in some applications, for example, as food ingredients to add typical mushroom flavour to different dishes. However, they can also be an obstacle in some others by introducing strong profile of mushroom aroma in food and personal care products developed from bioactive compounds in mushrooms such as polysaccharides, ${ }^{2,7}$ phenolic compounds, ${ }^{2,7,16}$ sterols and triterpenoids. ${ }^{2,7}$ For example, mushroom aroma in food without mushrooms is important for the customers, who do not accept the structure and taste of mushrooms, while for cosmetic products, ${ }^{16}$ bioactive ingredients or extracts from mushrooms shall be free of the aroma/smell of mushrooms. In both view, it 
is worthwhile to separate aroma compounds from mushrooms, yielding volatile compounds which could be used as food ingredients, and aroma-free residue, which can be further extracted to obtain bioactive compounds (sterols and polysaccharide) without mushroom smell.

On the other hand, hydrodistillation (HD) and organic solvent extraction, such as simultaneous distillation extraction (SDE) using a Likens-Nickerson instrument, ${ }^{17}$ are traditional processes used for the extraction of essential oils from aromaactive and medicinal plants. ${ }^{9,15,18}$ Nevertheless, these extraction methods are time consuming, and some compounds are susceptible to chemical changes under high temperatures. ${ }^{19}$ Additionally, some volatile compounds could also be lost during the solvent removal. ${ }^{20}$ Supercritical fluid extraction (SFE) is a suitable method to extract valuable components from natural raw materials. SFE offers features that overcome many limitations of conventional extraction methods, thus, being a suitable alternative to conventional processes such as HD, SDE, and organic solvent extraction. ${ }^{21}$ Furthermore, high extraction yield and extract quality could be achieved using optimized supercritical fluid extraction parameters. ${ }^{22,23}$ Supercritical carbon dioxide $\left(\mathrm{SC}-\mathrm{CO}_{2}\right)$ is the most preferred and commonly used supercritical fluid because it is non-toxic, chemically stable, environmental friendly, and easy to be removed from the extract yielding a solvent-free extract. ${ }^{21,23}$ It also has the potential for selective and efficient extraction by controlling the pressure and temperature, which regulate the density and solvating power of $\mathrm{CO}_{2} \cdot{ }^{24-27}$ Thus, volatiles can be selectively extracted from mushrooms, and other compounds, such as protein, polysaccharides and sterols can be well protected in the residue and have the possibility for further application. Many researchers have reported the extraction of mushroom aroma compounds based on HD or SDE. ${ }^{\mathbf{9 , 1 3 - 1 5 , 2 0 , 2 8 , 2 9}}$ Previous works related to supercritical fluid extraction of mushrooms focused on biologically active compounds including fatty acids and sterols as targets of extraction; ${ }^{30-32}$ the characteristic of extracts and the effect of extraction parameters on the total extraction yield was further studied. However, there are very limited publication on the SFE of volatile compounds from mushrooms and the impact of SFE parameters on the yield and composition of extracted volatile compounds.

Partial least-squares regression (PLSR) has been effectively used to explain the correlation of variables by obtaining information from raw data and focusing on a comprehensive evaluation of the obtained information. ${ }^{33,34}$ PLSR analysis method facilitates to create models for accurate prediction of the chemical characteristics of unknown food samples. ${ }^{35}$ The aroma compounds or/and bioactive components can be selectively extracted by supercritical fluid extraction for further characterization. Combining extraction parameters with GCFID results in PLSR analysis, prediction models can be built up based on the correlation between SFE parameters and the yield of volatile compounds in the extracts. Such models could be an effective tool for ensuring efficient selective extraction of typical volatile compounds from Craterellus tubaeformis and other mushrooms.
This study determined the optimal range of parameters for extracting volatiles from dried Finnish wild mushrooms (Craterellus tubaeformis) and studied the composition of the extracts and the content of each extracted volatile compound under different extraction parameters. The effect of extraction parameters on extraction yield and the content of mushroom alcohols were also evaluated to assess the supercritical fluid's potential for selective and efficient extractions of major aroma components. Additionally, the correlation between chemical profiles and supercritical fluid extraction parameters were analysed with PLSR to design models for the prediction of volatile contents in mushroom extracts under different SFE operating parameters. The proposed prediction models will be helpful to optimize the processing conditions of SFE for extracting aroma compounds from Finnish wild mushrooms as well as other wild mushrooms. This study provides guidance and theoretical reference for commercial-scale supercritical fluid extraction to collect target volatile compounds from mushrooms as ingredients for food flavouring and fragrance, meanwhile yielding aroma-free residues for special purpose.

\section{Materials and methods}

\subsection{Mushroom material and reagents}

Frozen Finnish wild mushroom Craterellus tubaeformis was purchased from Sieneste Oy (Joensuu, Finland). The mushroom samples were dried for five hours in a ventilated hot air-drying oven at $45{ }^{\circ} \mathrm{C}$, then grinded to obtain mushroom powder (1.0 mm blade mesh was used to control the particle size of mushroom powder). The mushroom powders were then stored at $4{ }^{\circ} \mathrm{C}$. The reference compounds of benzaldehyde, 1-octen-3ol, 2-octen-1-ol, linalool, and nonanal were purchased from Sigma-Aldrich. $p$-Xylene as internal standard was purchased from Fluka Chemicals (GmbH CH-9471 Buchs). A mixture of hydrocarbons ranging from octane (C8) to tetracontane (C40) was purchased from AccuStandard Inc. (New Haven, USA). Hexane and methanol of HPLC grade were purchased from VWR International S. A. S. (France). All reagents were of analytical grade unless otherwise stated.

\subsection{Supercritical fluid extraction procedures and experimental design}

Supercritical $\mathrm{CO}_{2}$ was used for extracting aroma compounds from mushrooms using a laboratory-scale SFX220 dynamic extraction system (ISCO, Lincoln, NE, USA) with a maximum pressure of 700 bar and a maximum temperature of $150{ }^{\circ} \mathrm{C}$ as shown in Fig. 1. Approximately $6 \mathrm{~g}$ of mushroom powder were weighted and put into a $10 \mathrm{~mL}$ extractor cell, which was placed in the ISCO extraction chamber and allowed to equilibrate to the set extraction temperature. $10 \mathrm{~mL}$ hexane was put in the collecting tube to absorb extracted volatile compounds. A glass wool was inserted at the top of the collecting tube and the tube was placed in an ice bath to reduce the loss of volatile compounds. Immediately after the extraction, the extracts were transferred into $2 \mathrm{~mL}$ vials for further analysis. The density of $\mathrm{CO}_{2}$ mentioned below was obtained from online-calculation- 


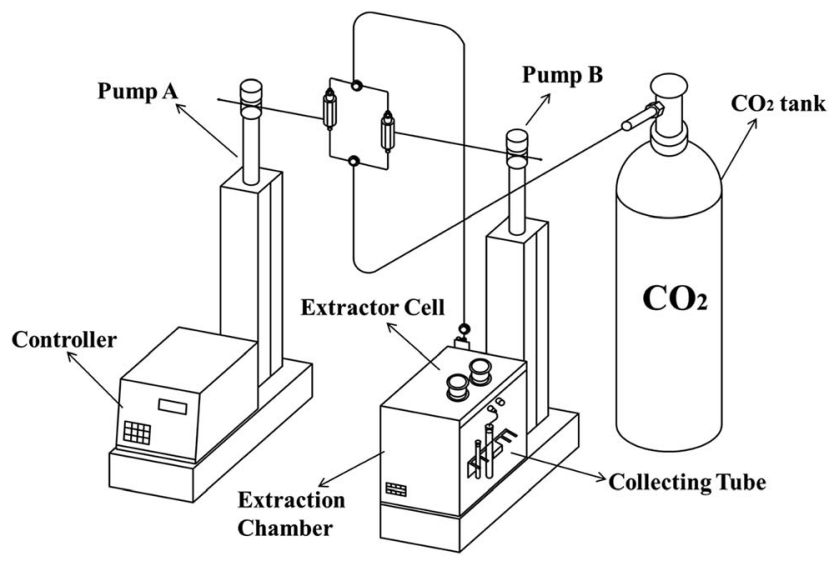

Fig. 1 Diagram of the supercritical fluid extraction apparatus.

carbon dioxide, ${ }^{36}$ using the calculation of thermodynamic state variables of carbon dioxide, with lower limit for calculation of $-55{ }^{\circ} \mathrm{C}, 1 \mathrm{bar}$ and upper limit of $900{ }^{\circ} \mathrm{C}, 1000 \mathrm{bar}$. Single factor experiments were performed to evaluate the effect of temperature, pressure and $\mathrm{SC}-\mathrm{CO}_{2}$ volume on the total extraction yield and the selectivity of $\mathrm{SC}-\mathrm{CO}_{2}$ to the mushroom-alcohol compounds. The extraction efficiency generally depends on the flow rate of $\mathrm{CO}_{2}$ and extraction time, however the $\mathrm{CO}_{2}$ flow rate could not be adjusted for SFX220 dynamic extraction system. Therefore, the $\mathrm{SC}-\mathrm{CO}_{2}$ volume was used as a comprehensive parameter to reflect the combined effect of $\mathrm{CO}_{2}$ flow rate and extraction time. The following extraction parameters, pressure (80, 85, 90 and 95 bar), temperature $(35,40,45,50$ and $55{ }^{\circ} \mathrm{C}$ ) and $\mathrm{SC}^{-\mathrm{CO}_{2}}$ volume (30, 50, 60 and $\left.70 \mathrm{~mL}\right)$ were independent variables during the test. To further study the effect of operating parameters on the composition of the extracts, an orthogonal-array design based on the single factor experiments was used, which can maximize the test coverage while minimizing the number of test cases. All the supercritical extraction parameters are shown in Table 1. These experiments were used further for models development described below.

\subsection{Extraction yield measurement}

The extracted aroma compounds were absorbed by hexane and it was hard to know their exact amount, therefore the extraction

Table 1 Experimental design

\begin{tabular}{llll}
\hline \multirow{4}{*}{ No. } & Factors & & \\
\cline { 2 - 4 } & Temperature $/{ }^{\circ} \mathrm{C}$ & Pressure/bar & SC-CO $\mathrm{CO}_{2}$ volume $/ \mathrm{mL}$ \\
\hline 1 & 40 & 85 & 30 \\
2 & 40 & 90 & 50 \\
3 & 40 & 95 & 70 \\
4 & 45 & 90 & 70 \\
5 & 45 & 95 & 30 \\
6 & 45 & 85 & 50 \\
7 & 50 & 95 & 50 \\
8 & 50 & 85 & 70 \\
9 & 50 & 90 & 30
\end{tabular}

cell was weighed before and after extraction to obtain a rough estimation of extraction yield using the following equation:

$$
\text { Total extraction yield } \%=\frac{m_{\mathrm{b}}-m_{\mathrm{a}}}{m_{\mathrm{b}}-m_{\mathrm{e}}}
$$

where $m_{\mathrm{b}}$ and $m_{\mathrm{a}}$ represent the mass of extraction cell loaded with the sample powder before and after extraction, $m_{\mathrm{e}}$ is the mass of the empty extraction cell.

\subsection{GC-FID and GC-MS analysis of extracted compounds}

The mushroom extracts were analysed using a Shimadzu GC2010 Auto Injector/Auto Sampler gas chromatograph (GC) equipped with a flame ionisation detector (FID). A DB-1 fused silica capillary column (30 m length, $0.25 \mathrm{~mm}$ inner diameter, $0.25 \mu \mathrm{m}$ film thickness, Agilent Technologies, USA) was used. $1 \mu \mathrm{L}$ sample was injected automatically via a split/splitless injector. Helium was applied as the carrier gas at a constant flow rate of $1.2 \mathrm{~mL} \mathrm{~min}^{-1}$, and the oven temperature was programmed from $40{ }^{\circ} \mathrm{C}$ (held for $15 \mathrm{~min}$ ) to $300{ }^{\circ} \mathrm{C}$ at the rate of $9{ }^{\circ} \mathrm{C} \mathrm{min}{ }^{-1}$, held for $2 \mathrm{~min}$ at $300{ }^{\circ} \mathrm{C}$. Additionally, the extracts were analysed by a Hewlett Packard HP 6890 GC/MS system using the same column as in GC-FID analysis. The mass spectrometer was operated in electron ionization (EI) mode at $70 \mathrm{eV}$, and the flow rate of the carrier gas and the column temperature programme were the same as those of GC-FID analysis.

A series of $n$-alkanes (C8-C40) was analysed under the same conditions to obtain the linear retention index (RI). The RI was calculated according to the following equation:

$$
\mathrm{RI}=100 \times\left(n+(N-n) \frac{\operatorname{TR}(x)-\mathrm{TR}(n)}{\mathrm{TR}(N)-\mathrm{TR}(n)}\right)
$$

where TR is the retention time, $N$ and $n$ are the numbers of carbon atoms in the alkanes, which were eluted after and before the compound $x$, respectively.

The quantities of volatiles were calculated by comparison of their peak areas with that of the internal standard. The peaks of the volatile compounds were identified by both mass spectrum and retention index; also, some authentic compounds were used for the volatiles identification.

\subsection{Data analysis}

2.5.1 Partial least squares regression (PLSR) analysis. Partial least squares regression (PLSR) analysis was carried out using Unscrambler version X 10.4 (CAMO ASA, Oslo, Norway). Based on the experimental design (single factor experiments and orthogonal-array designed experiments) mentioned above, 54 experimental sets (with varying extraction parameters, temperature from 35 to $55{ }^{\circ} \mathrm{C}$, pressure from 80 bar to 95 bar and $\mathrm{SC}-\mathrm{CO}_{2}$ flow volume from 30 to $70 \mathrm{~mL}$ for each experimental set) were done including duplication experiments. And they were divided randomly into thirty experimental sets and twenty-four experimental sets. The thirty experimental sets were used to evaluate the correlation between SFE parameters and the relative content i.e. extraction yield $(\mu \mathrm{g} / 100 \mathrm{~g}$ mushroom samples) of extracted aroma compounds using PLS2 methods (PLSR performed using many $X$-variables and several $Y$-variables 
simultaneously). Additionally, with these thirty experimental sets, model calibration was performed using PLS1 (PLSR performed using many $X$-variables and only one $Y$-variable) by a jack-knifing test. The twenty-four experimental sets were used to check the predictive capability of developed models. All $Y$ variables were centered and standardized (1/SDev) to obtain the unbiased contribution of each variable, regardless of whether they have a small or large standard deviation from the outset. Significance level was set at $p<0.05$.

2.5.2 Statistical analysis. Statistical analysis was performed using Microsoft Excel 2013 (Microsoft, Redmond, WA USA) and SPSS 13.0 (IBM, Armonk, NY USA). The mean \pm standard deviation (SD) values of mushroom volatile compounds were reported and differences reaching $p<0.05$ were considered as statistically significant.

\section{Results and discussion}

Dried mushrooms was used in our study mostly because drying is a common step of mushroom processing and preserving; thus using dried mushroom can make our study have more practical significance; moreover, the drying procedure operated under relative higher temperature may contribute to the oxidation of unsaturated fatty acid in Craterellus tubaeformis, forming more volatile compounds, including aldehydes, ketones and alcohols which can make the aroma profile of mushrooms more saturated and complete. ${ }^{\mathbf{1 0}}$ Additionally, the excess water in the sample will act as a barrier in transfer of the analytes to the supercritical $\mathrm{CO}_{2}$ fluid during SFE procedure ${ }^{21,22}$ and drying can reduce the high water content in fresh mushrooms.

This research is part of a larger project aimed for comprehensive utilization of the wild mushroom (Craterellus tubaeformis) resource by multi-stage extraction and separation. Extracting volatile compounds and further correlating SFE parameters with the yield of volatiles is the first step of the whole project and the focus of the current manuscript. The aim is to selectively extract volatile compounds as flavour-enhancing food ingredients, whereas the aroma-free residues can be further extracted by SFE or other extraction methods to obtain fatty acids, polysaccharides and protein for the study of functional properties or the development of novel products. The latter part was not the focus of the present work. Sample pretests were performed to determine the optimal range of extraction parameters for increasing the yield of volatile compounds from mushrooms, meantime avoiding coextraction of fatty components. During the pre-tests, maintaining the $\mathrm{SC}-\mathrm{CO}_{2}$ density between 0.205 and $0.657 \mathrm{~g} \mathrm{~mL}^{-1}$ (pressure from 80 to 95 bar, temperature from 35 to $55^{\circ} \mathrm{C}$ ) was sufficient for the extraction of desirable volatile compounds, such as 1-octen-3-ol, limonene, 2-octen-1-ol and nonanal, from mushrooms without fatty acids. Thus, single factor experiments were done based on parameters within these ranges. Similar findings were reported by Bocevska et al. ${ }^{37}$ who found that the moderate extraction conditions $\left(\mathrm{SC}-\mathrm{CO}_{2}\right.$ density of $0.290 \mathrm{~g} \mathrm{~mL}^{-1}$ under the pressure of $100 \mathrm{bar}$ and the temperature of $60{ }^{\circ} \mathrm{C}$ ) were the most selective for limonene extraction from yarrow flowers with respect to unwanted waxes.

\subsection{Influence of SFE operating parameters}

The results of SFE can be influenced by different parameters during extraction process including temperature, pressure and $\mathrm{SC}-\mathrm{CO}_{2}$ volume. The effect of temperature, pressure and $\mathrm{SC}-\mathrm{CO}_{2}$ volume on extraction yield was studied and the content of mushroom alcohol compounds were measured. The mushroom extracts were qualitative analysed by GC-MS and further quantified using GC-FID based on the internal standard. The results showed that 1-octen-3-ol and 2-octen-1-ol were the only two typical mushroom alcohols identified in the samples. Their content (\%) in the extract was used to reflect the selectivity of the $\mathrm{SC}-\mathrm{CO}_{2}$ on mushroom alcohol compounds.

The influence of pressure was studied from 80 bar to 95 bar, with the temperature set at $40{ }^{\circ} \mathrm{C}$ and the $\mathrm{SC}-\mathrm{CO}_{2}$ volume at $50 \mathrm{~mL}$. Results showed that the total extraction yield was enhanced as the extraction pressure increased from 85 to 95 bar with the highest yield of extract reaching $0.76 \%$ (Fig. 2a). This was because the elevation of pressure at fixed temperature resulted in an increase of the density of $\mathrm{SC}-\mathrm{CO}_{2}$ from 0.278 to $0.557 \mathrm{~g} \mathrm{~mL}^{-1}$, which led to the enhancement of the solvent power of SC- $\mathrm{CO}_{2}$. Additionally, the increased density of $\mathrm{SC}-\mathrm{CO}_{2}$ might also have accelerated the mass transfer between the analytes and solvent during extraction process, therefore, improving the total extraction yield. ${ }^{22,23}$ These findings were in accordance with previously reported studies on the SFE of substances from mint leaves (Mentha spicata), ${ }^{38}$ Bulgarian Achillea millefolium, ${ }^{39}$ quinoa (Chenopodium quinoa willd) seeds, ${ }^{\mathbf{4 0}}$ Zingiber officinale var. Amarum, ${ }^{\mathbf{4 1}}$ coriander (Coriandrum sativum L., Apiaceae) seeds, ${ }^{\mathbf{2 6}}$ and common carp (Cyprinus
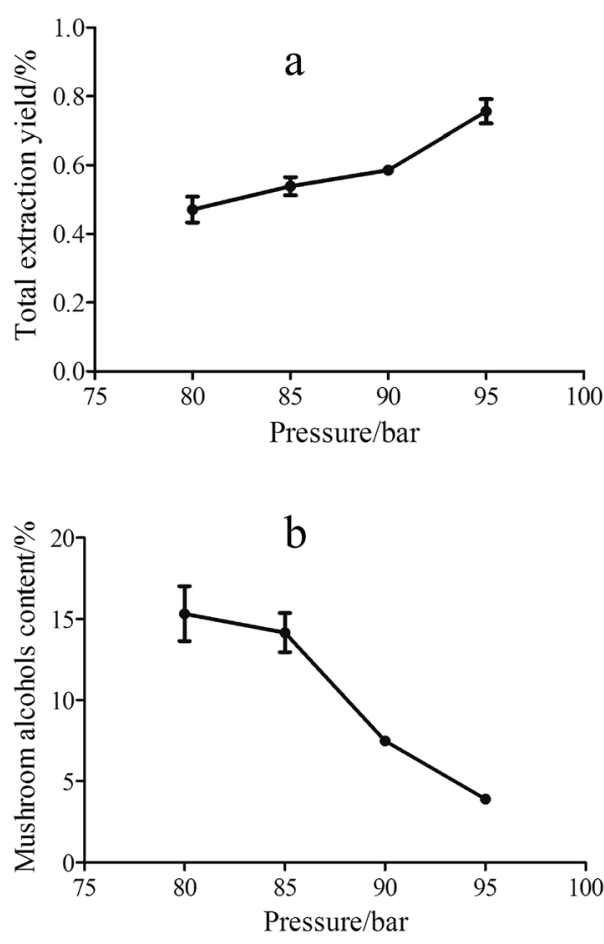

Fig. 2 Influence of pressure on total extraction yield (a) and the content of mushroom alcohols (b) at $80-95$ bar, $40{ }^{\circ} \mathrm{C}, 50 \mathrm{~mL} \mathrm{SC}$ $\mathrm{CO}_{2}$. 
carpio L.). ${ }^{42}$ As shown in Fig. $2 \mathrm{~b}$, the content of mushroom alcohols decreased from $15 \%$ to $3 \%$ with the increase of pressure. This might be due to the higher solvating power of $\mathrm{SC}-\mathrm{CO}_{2}$, which decreased the extraction selectivity and increased the coextraction of non-volatile compounds. ${ }^{22}$ Thus, the presence of co-extracted solutes under higher pressure reduced the extraction efficiency of the mushroom-alcohol compounds. Similar observations were reported by Hamburger et al. ${ }^{\mathbf{4 3}}$ that at higher pressure some non-volatile lipophilic compounds were coextracted with target substances. Hence, taking these two figures (Fig. 2a and b) into account, 85 bar might be the best pressure for extracting mushroom alcohols because of its satisfied total extraction yield and relatively desirable extract.

Moreover, the effect of temperature has been also evaluated in the range between 35 to $55{ }^{\circ} \mathrm{C}$ while the pressure and $\mathrm{SC}-\mathrm{CO}_{2}$ was kept at 85 bar and $50 \mathrm{~mL}$, respectively. As shown in Fig. 3, the temperature influenced both the total extraction yield and the mushroom alcohols content. With the increase of temperature, the density of $\mathrm{CO}_{2}$ and the corresponding solvent strength decreased, which had a negative effect on the total extraction yield. On the other hand, the elevated temperature also increased vapor pressure of the volatile compounds, facilitating the extraction of these compounds. For extracting aroma compounds under different temperatures, there is a competition between the solubility of $\mathrm{SC}-\mathrm{CO}_{2}$ (which decreases with increasing temperature) and the vapor pressure of extracted compounds (which rises with increasing temperature) ${ }^{22,42}$ As shown in Fig. 3a, the increasing temperature from 35 to $55{ }^{\circ} \mathrm{C}$
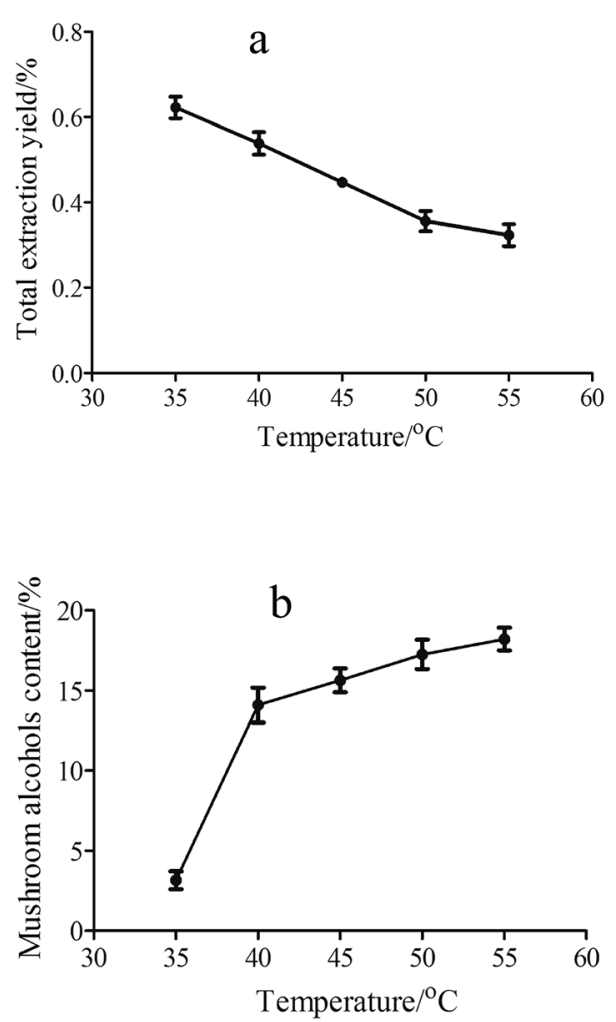

Fig. 3 Influence of temperature on total extraction yield (a) and the content of mushroom alcohols (b) at 85 bar, $35-55^{\circ} \mathrm{C}, 50 \mathrm{~mL} \mathrm{SC}-\mathrm{CO}_{2}$. decreased the total extraction yield from $0.6 \%$ to $0.32 \%$, which was mostly due to the weak solubility of $\mathrm{SC}-\mathrm{CO}_{2}$ at lower density despite the enhanced solute sublimation at higher temperature. Similar findings were reported by Zekovic et al. ${ }^{\mathbf{2 6}}$ and Salea et $a .^{\mathbf{4 1}}$ using supercritical fluid to extract substances from coriander seeds and Zingiber officinale var. Amarum, respectively. Moreover, for the content of mushroom alcohol compounds, there was a sharp increase from $35{ }^{\circ} \mathrm{C}$ to $40{ }^{\circ} \mathrm{C}$ (Fig. 3b) and followed by a slight increase from 40 to $55^{\circ} \mathrm{C}$. The decreased solvent strength of $\mathrm{SC}-\mathrm{CO}_{2}$ increased the extraction selectivity, avoiding undesired co-extraction of non-volatile compounds, such as fatty acids and other lipids. In addition, the volatile compounds were extracted more easily because of their enhanced vapor pressure, therefore, the content of mushroom alcohol compounds in the extracts increased. Thus, $40{ }^{\circ} \mathrm{C}$ was selected as the optimum temperature for extracting mushroom alcohols, providing acceptable total extraction yield and higher content of the target compounds.

The larger amount of fluid volume means longer extraction time and likely more sufficient contact between the supercritical fluid and the material samples. Fig. 4 shows the effect of SC$\mathrm{CO}_{2}$ volume on total extraction yield and content of mushroom alcohols. From Fig. 4a it was observed that the total extraction yield increased from $0.43 \%$ to $0.60 \%$ with increasing $\mathrm{SC}-\mathrm{CO}_{2}$ volume from 30 to $60 \mathrm{~mL}$, and after $60 \mathrm{~mL}$ the yield increase tended to be gentle, which indicated that $60 \mathrm{~mL}$ was enough for volatiles extraction at 85 bar and $40{ }^{\circ} \mathrm{C}$. Moreover, a decrease trend of mushroom alcohols content was seen from 30 to $60 \mathrm{~mL}$
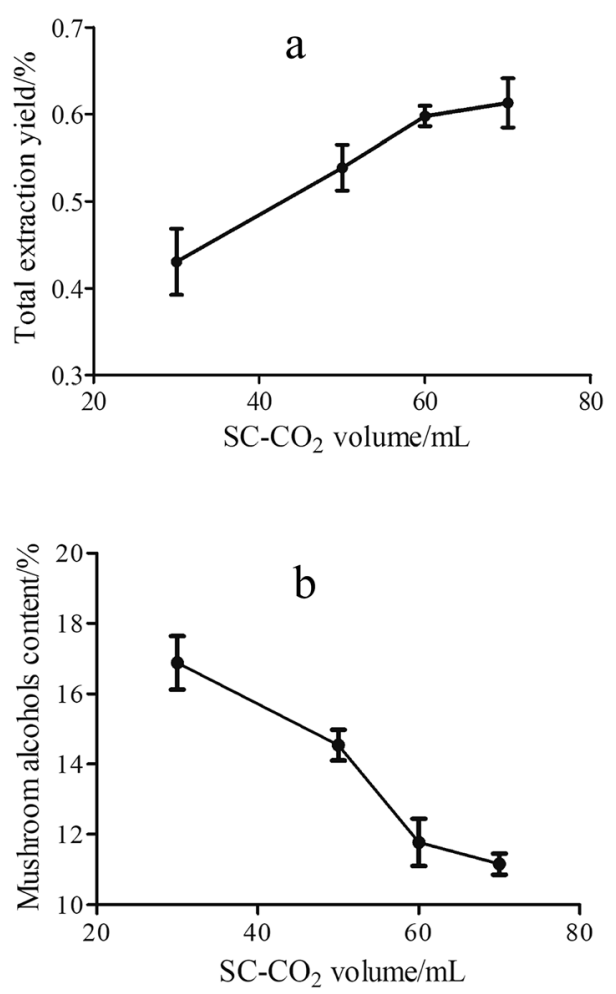

Fig. 4 Influence of $\mathrm{SC}-\mathrm{CO}_{2}$ volume on total extraction yield (a) and the content of mushroom alcohols (b) at 85 bar, $40^{\circ} \mathrm{C}, 30-70 \mathrm{~mL} \mathrm{SC}$ $\mathrm{CO}_{2}$ 
SC- $\mathrm{CO}_{2}$ (Fig. 4b), and a relative low content was obtained at $60 \mathrm{~mL} \mathrm{SC}-\mathrm{CO}_{2}$; these results suggested that with prolonged extraction time, the co-extraction of unwanted compounds occurred, which reduced alcohols content (eight carbon alcohols). Similar findings were reported by Kitzberger et al. ${ }^{30}$ who found that the extraction time affected the extract composition when extracting oil from shiitake; moreover, low molecular weight compounds tended to be extracted in the beginning of extraction process, followed by more polar compounds. Finally, taking the extraction yield and the content of mushroom alco-

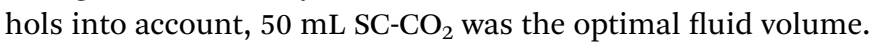

\subsection{Analysis of extracted volatile compounds by GC and GC/ MS}

The extraction pressure and temperature affect the density of $\mathrm{CO}_{2}$ and vapor pressure of compounds, which directly influence and modulate the solubility of volatile compounds in supercritical $\mathrm{CO}_{2}$, resulting in different chemical profile under various extraction conditions. An orthogonal-array experiment was designed (Table 1) based on the results of the single factor experiments to study the impact of the extraction parameters on the yield of extracted volatile compounds. Mushroom extracts were quantified by GC-FID. More than 50 peaks have been identified, however, only 17 peaks were in common and most of these common extracted compounds play important roles in the overall aroma of mushrooms, such as 1-octen-3-ol (mushroomlike), ${ }^{44}$ 2-octen-1-ol (mushroom-like), ${ }^{44}$ 6-methyl-5-hepten-2-one (green, mushroom-like), ${ }^{\mathbf{4 5}}$ limonene (lemon-like), ${ }^{\mathbf{4 4}}$ nonanal (fruity, sweet, pine-like), ${ }^{45}$ linalool (citrus-like), ${ }^{44}$ and benzaldehyde (burnt sugar, almond). ${ }^{45}$ The relative contents of these 17 common extracted aroma compounds are presented in Table 2.

As shown in Table 2, 1-octen-3-ol and 2-octen-1-ol were the only two mushroom-alcohol compounds extracted from the samples. The highest yield of 1-octen-3-ol (360 $\mu \mathrm{g} / 100 \mathrm{~g})$ was found in experiment $8\left(85 \mathrm{bar}, 50{ }^{\circ} \mathrm{C}, 70 \mathrm{~mL} \mathrm{SC}-\mathrm{CO}_{2}\right)$, while the highest amount of 2-octen-1-ol (290 $\mu \mathrm{g} / 100 \mathrm{~g})$ was obtained in experiment 3 (95 bar, $40{ }^{\circ} \mathrm{C}, 70 \mathrm{~mL}$ SC- $\mathrm{CO}_{2}$ ). A similar yield of 1octen-3-ol as in experiment 8 was also obtained in experiment 3 (95 bar, $40{ }^{\circ} \mathrm{C}, 70 \mathrm{~mL} \mathrm{SC}-\mathrm{CO}_{2}$ ) and experiment 4 (90 bar, $45^{\circ} \mathrm{C}$, $70 \mathrm{~mL}$ SC- $\left.\mathrm{CO}_{2}\right)$. Moreover, the highest yields of linalool $(290 \mu \mathrm{g} /$ $100 \mathrm{~g})$, geranyl acetone $(174 \mu \mathrm{g} / 100 \mathrm{~g})$, and bornyl acetate (128 $\mu \mathrm{g} / 100 \mathrm{~g}$ ) were all obtained in experiment $3\left(95 \mathrm{bar}, 40{ }^{\circ} \mathrm{C}, 70 \mathrm{~mL}\right.$ SC-CO $\left.{ }_{2}\right) \cdot N$-(3-Methylbutyl)acetamide, which was synthesized by Maillard reaction during the drying process, ${ }^{45}$ also showed the highest yield $(1590 \mu \mathrm{g} / 100 \mathrm{~g})$ in experiment 3 .

The structural characteristics of the compounds influence their solubility in supercritical $\mathrm{CO}_{2}$, resulting in different content for each compound under different extraction conditions. ${ }^{46}$ As reported by Zeković et al. ${ }^{26}$ about monoterpenes extraction from coriander seeds. Although limonene and terpinene possess the same chemical formula $\left(\mathrm{C}_{10} \mathrm{H}_{16}\right)$, the different position of the double bond in the molecule resulted in different solubility under the same extraction conditions. ${ }^{26}$ On the other hand, branching structures help to increase the solubility of alcohols, thus, secondary and tertiary alcohols have

Table 2 Qualitative and quantitative analysis of extracted volatile compounds

Content in sample ${ }^{d}(\mu \mathrm{g} / 100 \mathrm{~g}$ dried mushrooms)

\begin{tabular}{|c|c|c|c|c|c|c|c|c|c|c|c|c|}
\hline $\mathrm{RI}^{a}$ & $\mathrm{KI}^{b}$ & $\mathrm{ID}^{c}$ & Compound & 1 & 2 & 3 & 4 & 5 & 6 & 7 & 8 & 9 \\
\hline 738 & 757 & $\mathrm{AB}$ & 3-Hexanone & $29 \pm 2$ & $33 \pm 8$ & $27 \pm 1$ & $36 \pm 8$ & $29 \pm 3$ & $40 \pm 3$ & $43 \pm 7$ & $47 \pm 4$ & $40 \pm 1$ \\
\hline 765 & 785 & $\mathrm{AB}$ & 1-Octene & $36 \pm 5$ & $54 \pm 6$ & $117 \pm 8$ & $97 \pm 7$ & $60 \pm 8$ & $56 \pm 4$ & $87 \pm 2$ & $68 \pm 5$ & $32 \pm 2$ \\
\hline 791 & 819 & $\mathrm{AB}$ & 1,3-Octadiene & $230 \pm 20$ & $190 \pm 10$ & $190 \pm 10$ & $187 \pm 6$ & $180 \pm 10$ & $210 \pm 20$ & $203 \pm 3$ & $230 \pm 10$ & $190 \pm 20$ \\
\hline 898 & 926 & $\mathrm{ABC}$ & Benzaldehyde & $24 \pm 1$ & $34 \pm 2$ & $42 \pm 2$ & $42 \pm 4$ & $38 \pm 7$ & $36 \pm 6$ & $45 \pm 0$ & $46 \pm 4$ & $34 \pm 4$ \\
\hline 952 & 966 & $\mathrm{AB}$ & $\begin{array}{l}\text { 6-Methyl-5- } \\
\text { hepten-2-one }\end{array}$ & $42 \pm 4$ & $33 \pm 3$ & $36 \pm 3$ & $53 \pm 7$ & $50 \pm 8$ & $50 \pm 10$ & $56 \pm 3$ & $65 \pm 5$ & $53 \pm 8$ \\
\hline 954 & 961 & $\mathrm{ABC}$ & 1-Octen-3-ol & $290 \pm 10$ & $318 \pm 6$ & $350 \pm 10$ & $350 \pm 20$ & $280 \pm 10$ & $318 \pm 6$ & $298 \pm 8$ & $360 \pm 10$ & $260 \pm 10$ \\
\hline 975 & 980 & $\mathrm{AB}$ & 2-Pentylfuran & $74 \pm 4$ & $154 \pm 3$ & $236 \pm 5$ & $190 \pm 20$ & $120 \pm 20$ & $106 \pm 2$ & $140 \pm 10$ & $150 \pm 10$ & $64 \pm 3$ \\
\hline 994 & 1020 & $\mathrm{AB}$ & Limonene & $29 \pm 2$ & $32 \pm 2$ & $35 \pm 3$ & $36 \pm 2$ & $28 \pm 3$ & $31 \pm 0$ & $30 \pm 4$ & $35 \pm 5$ & $27 \pm 1$ \\
\hline 1027 & 1039 & $\mathrm{ABC}$ & 2-Octen-1-ol & $90 \pm 10$ & $180 \pm 4$ & $290 \pm 10$ & $180 \pm 2$ & $120 \pm 20$ & $120 \pm 10$ & $140 \pm 20$ & $130 \pm 10$ & $56 \pm 4$ \\
\hline 1050 & 1082 & $\mathrm{ABC}$ & Nonanal & $43 \pm 3$ & $61 \pm 3$ & $80 \pm 3$ & $54 \pm 2$ & $45 \pm 4$ & $34 \pm 5$ & $41 \pm 3$ & $34 \pm 4$ & $20 \pm 4$ \\
\hline 1062 & 1086 & $\mathrm{ABC}$ & Linalool & $110 \pm 10$ & $235 \pm 4$ & $290 \pm 20$ & $210 \pm 20$ & $140 \pm 10$ & $111 \pm 7$ & $140 \pm 10$ & $127 \pm 4$ & $60 \pm 6$ \\
\hline 1071 & - & $\mathrm{AB}$ & $\begin{array}{l}N \text {-(3-Methylbutyl)- } \\
\text { acetamide }\end{array}$ & $620 \pm 90$ & $1000 \pm 20$ & $1590 \pm 40$ & $1010 \pm 20$ & $750 \pm 40$ & $370 \pm 20$ & $480 \pm 20$ & $240 \pm 50$ & $154 \pm 6$ \\
\hline 1095 & 1088 & $\mathrm{AB}$ & $\begin{array}{l}\text { 6-Methyl-3,5- } \\
\text { heptadien-2-one }\end{array}$ & $65 \pm 2$ & $161 \pm 6$ & $179 \pm 6$ & $170 \pm 20$ & $120 \pm 10$ & $122 \pm 5$ & $150 \pm 20$ & $170 \pm 20$ & $110 \pm 20$ \\
\hline 1197 & 1193 & $\mathrm{AB}$ & 2,4-Nonadienal & $160 \pm 30$ & $240 \pm 30$ & $260 \pm 10$ & $250 \pm 10$ & $180 \pm 20$ & $180 \pm 10$ & $230 \pm 30$ & $200 \pm 20$ & $130 \pm 10$ \\
\hline 1265 & 1273 & $\mathrm{AB}$ & Bornyl acetate & $44 \pm 2$ & $100 \pm 10$ & $128 \pm 2$ & $86 \pm 5$ & $72 \pm 6$ & $50 \pm 6$ & $73 \pm 9$ & $55 \pm 6$ & $33 \pm 4$ \\
\hline 1284 & 1280 & $\mathrm{AB}$ & 2,4-Decadienal & $29 \pm 3$ & $106 \pm 0$ & $148 \pm 3$ & $88 \pm 9$ & $66 \pm 7$ & $33 \pm 2$ & $68 \pm 8$ & $41 \pm 5$ & $23 \pm 4$ \\
\hline 1405 & 1428 & $\mathrm{AB}$ & Geranyl acetone & $53 \pm 4$ & $139 \pm 3$ & $174 \pm 4$ & $127 \pm 8$ & $109 \pm 8$ & $68 \pm 7$ & $116 \pm 5$ & $74 \pm 5$ & $54 \pm 1$ \\
\hline
\end{tabular}

${ }^{a}$ RI means the Kovats index which were determined by a series of hydrocarbons (C8-C40) on the column of DB-1 described in Section $2.4{ }^{b}$ KI denotes the Kovats index reference from NIST standard reference database, by which the compositions were determined on a non-polar (HP/ DB-5 or HP/DB-1) column run under similar GC-FID conditions. ${ }^{c}$ The identification was indicated by the following symbols: (A) mass spectrum; (B) comparison between RI and KI; (C) authentic compounds. ${ }^{d}$ All GC peak areas were quantified as the internal standard ( $p$-xylene) and approximate concentrations (mean \pm standard deviation, average of triple samples) for the individual volatile compounds were shown in the table. 
better solubility than primary alcohol. ${ }^{46}$ In this study, the two mushroom alcohols, 1-octen-3-ol and 2-octen-1-ol, possess the same chemical formula $\left(\mathrm{C}_{8} \mathrm{H}_{16} \mathrm{O}\right)$ but differ in position of their hydroxyl group and double bond resulting in different solubility in $\mathrm{SC}-\mathrm{CO}_{2}$. During the extraction, the density of $\mathrm{SC}-\mathrm{CO}_{2}$ was ranged from 0.252 to $0.557 \mathrm{~g} \mathrm{~mL}^{-1}$ (calculated based on the temperature and pressure applied). However, the content of extracted 1-octen-3-ol did not show significant changes and this probably due to its high solubility in $\mathrm{SC}-\mathrm{CO}_{2}$. As a secondary alcohol, 1-octen-3-ol was more efficiently extracted compared with 2-octen-1-ol (primary alcohol), and the relative poor solubility of 2-octen-1-ol required higher density $\mathrm{SC}^{-\mathrm{CO}_{2}}$ (95 bar, $40{ }^{\circ} \mathrm{C}, 70 \mathrm{~mL} \mathrm{SC}-\mathrm{CO}_{2}$ ) to reach its highest yield.

\subsection{Correlation between extracted volatile compounds and supercritical fluid extraction parameters}

The PLSR model was applied to study the relationship between the supercritical fluid extraction parameters ( $X$-matrix) and the content of extracted volatile compounds ( $Y$-matrix). The PLSR model included three factors explaining most of cross-validated variance. Only factor 1 versus factor 2 was presented in Fig. 5 since other factors did not provide more information. Except the compound 1,3-octadiene, all variables of SFE parameters and extracted volatiles were located between the inner and outer ellipses, which denote $50 \%$ and $100 \%$ of variance, respectively, indicating that these variables were well explained by the PLSR model. SFE parameters marked with small circles represented variables which contributed significantly to most extracted volatile compounds $(p<0.05)$. Moreover, results showed that SC$\mathrm{CO}_{2}$ volume was positively correlated to most aroma compounds including 2,4-decadienal, 2-pentylfuran, bornyl acetate, 1octene, 2,4-nonadienal, geranyl acetone etc. Additionally, the temperature had significant but negative correlation with the yield of the most aroma compounds except 3-hexanone, benzaldehyde, 6-methyl-3,5-heptadien-2-one, 6-methyl-5-hepten-2one. While pressure significantly and positively correlated with most of volatiles except 1,3-octadiene (Fig. 5).

\subsection{Prediction of extracted volatile compounds by PLSR}

PLS1 analysis was applied to further investigate the predictability of extracted volatiles under varying supercritical fluid

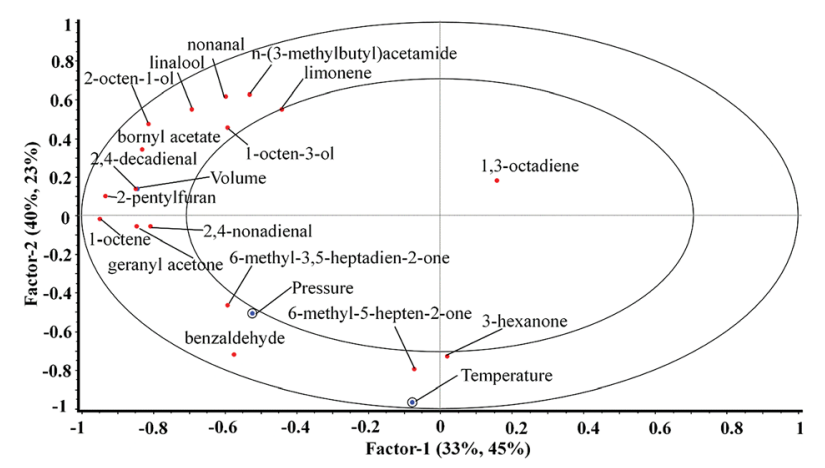

Fig. 5 Overview of the variables in the PLSR correlation loadings plot. parameters. Ten extracted volatiles, which dominated quantitatively among the extracted volatiles and represented the typical mushroom aroma compounds, ${ }^{\mathbf{4 4 , 4 5}}$ were chosen to design the mathematical models. The multiple regression curves as the predicted equations were established based on the calibration data sets. The yield of each extracted volatile compound ( $\mu \mathrm{g} / 100 \mathrm{~g}$ dried mushrooms) was assigned as $Y$ variable and the SFE parameters as predictive $X$-variables. The components of predicted equations are shown in Table 3. Predicted equations are described as $Y_{\text {volatile compounds }}=N_{1} X_{1}+$ $N_{2} X_{2}+N_{3} X_{3}+B_{0}$, where $Y_{\text {volatile compounds }}$ is the yield of the volatile compounds, and $X_{1}, X_{2}, X_{3}$ represent the $X$-variables pressure, temperature and SC- $\mathrm{CO}_{2}$ volume, respectively; $N_{1}, N_{2}$, $N_{3}$ denote the regression coefficients of the corresponding $X$ parameters, respectively.

Table 3 Components of predicted equations for typical volatile compounds extracted based on SFE parameters using PLS1 crossvalidation analysis ${ }^{a}$

\begin{tabular}{lllll}
\hline & \multicolumn{4}{l}{$\begin{array}{l}\text { Regression coefficients of the parameters }(X- \\
\text { variable) }\end{array}$} \\
\cline { 2 - 5 } $\begin{array}{l}\text { Mushroom volatiles } \\
\text { (Y-variable) }\end{array}$ & $N_{1}$ & $N_{2}$ & $N_{3}$ & $B_{0}$ \\
\hline 1-Octene & $2.827^{*}$ & -0.727 & $1.502^{*}$ & -226.291 \\
1-Octen-3-ol & $-1.329^{*}$ & $-1.694^{*}$ & $1.831^{*}$ & 421.447 \\
2-Pentylfuran & $5.267^{*}$ & $-2.753^{*}$ & $2.700^{*}$ & -351.193 \\
2-Octen-1-ol & $6.680^{*}$ & $-8.376^{*}$ & $2.777^{*}$ & -218.794 \\
Linalool & $7.212^{*}$ & $-10.345^{*}$ & $2.808^{*}$ & -170.586 \\
Nonanal & $1.722^{*}$ & $-2.609^{*}$ & $0.476^{*}$ & -17.597 \\
$N$-(3-Methylbutyl)- & $53.368^{*}$ & $-81.657^{*}$ & $11.470^{*}$ & -1004.286 \\
acetamide & & & & \\
Bornyl acetate & $3.855^{*}$ & $-3.621^{*}$ & $1.042^{*}$ & -167.499 \\
2,4-Decadienal & $6.047^{*}$ & $-3.723^{*}$ & $1.371^{*}$ & -383.743 \\
Geranyl acetone & $7.463^{*}$ & $-2.870^{*}$ & $1.376^{*}$ & -515.41
\end{tabular}

${ }^{a}$ The values marked with "*" denote that the SFE parameters were significant as a level of $p<0.05$.

Table 4 Predictive performance of developed equations

\begin{tabular}{lllll}
\hline & \multicolumn{4}{l}{ Statistical parameters } \\
\cline { 2 - 5 } Mushroom volatiles & $R_{\text {_cal }}{ }^{a}$ & $R_{\text {_cal }}^{2 b}$ & $R_{\text {_val }}{ }^{c}$ & $R_{- \text {val }}^{2 d}$ \\
\hline 1-Octene & 0.95 & 0.90 & 0.93 & 0.87 \\
1-Octen-3-ol & 0.89 & 0.79 & 0.86 & 0.74 \\
2-Pentylfuran & 0.94 & 0.88 & 0.92 & 0.85 \\
2-Octen-1-ol & 0.96 & 0.91 & 0.94 & 0.88 \\
Linalool & 0.90 & 0.82 & 0.89 & 0.78 \\
Nonanal & 0.93 & 0.86 & 0.90 & 0.80 \\
$N$-(3-Methylbutyl)acetamide & 0.92 & 0.85 & 0.90 & 0.81 \\
Bornyl acetate & 0.97 & 0.94 & 0.95 & 0.91 \\
2,4-Decadienal & 0.95 & 0.90 & 0.93 & 0.86 \\
Geranyl acetone & 0.96 & 0.92 & 0.95 & 0.90
\end{tabular}

${ }^{a} R_{\text {cal }}$ denotes the correlation coefficient of the data fit with the calibration model. ${ }^{b} R_{\text {cal }}{ }^{2}$ is the raw regression coefficient $\left(R^{2}\right)$ of the calibration model. ${ }^{c} R_{\text {val }}$ denotes the correlation coefficients of the data fit with the validation model. ${ }^{d} R_{\text {val }}{ }^{2}$ is the adjusted regression coefficients $\left(R^{2}\right)$ of the validation model. 
The predictive performance of these equations was estimated via the parameters of the fitted linear calibration and validated models (Table 4). Fitted linear calibration models showed that the correlation coefficient $\left(R_{-}\right.$cal $)$, represented by the regression model and the mean data, were greater than 0.90 , and the regression coefficients of the linear calibration models $\left(R_{-}{ }^{2}{ }^{2}\right)$ were greater than 0.82 for all the typical volatiles (except 1-octen-3-ol), indicating a good fit to the calibration model. The calibrated parameters for geranyl acetone $\left(R_{-\mathrm{cal}}=0.96 R_{-\mathrm{cal}}{ }^{2}=\right.$ $0.92)$ and 2-octen-1-ol $\left(R_{-}\right.$cal $\left.=0.96 R_{\text {_cal }}{ }^{2}=0.91\right)$ indicated that the mean data was excellent fitted to calibration model. However, the values of $R_{-}$cal $=0.89$ and $R_{-}$cal ${ }^{2}=0.79$ for 1 -octen3-ol suggested a slightly weaker fit compared with other compounds. The linear validated models were well fitted for 1octene, 2-pentylfuran, 2-octen-1-ol, bornyl acetate, 2,4- decadienal and geranyl acetone with correlation coefficients ( $R_{-}$val) greater than 0.91 , which means that the extraction yield of these compounds can be projected by the SFE parameters. On the other hand, regression coefficient of the linear validated equation $\left(R_{-}\right.$val $\left.{ }^{2}\right)$ used to check the adequacy of the model represents how successfully the cross-validated regression line approximates raw data points. ${ }^{47}$ As for most extracted typical volatiles, the $R_{- \text {val }}{ }^{2}$ were greater than 0.80 (except linalool and 1octen-3-ol), which suggested good predictive performance of the derived models.

\subsection{Validation of established prediction models}

Another independent data set including 24 samples was built in order to evaluate the validity of the established models. The
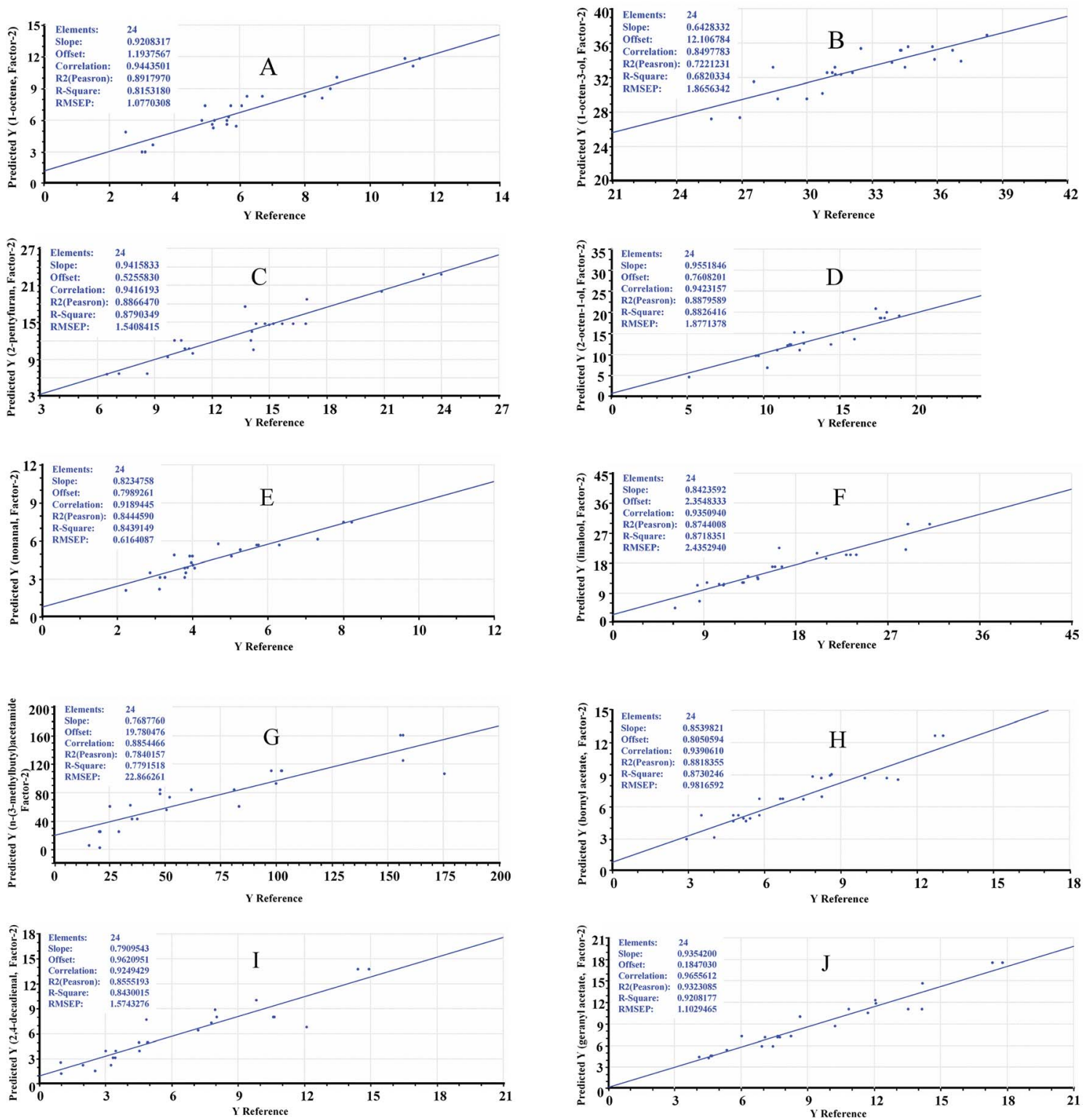

Fig. 6 Validation of established prediction models for 1-octene (A), 1-octen-3-ol (B), 2-pentyfuran (C), 2-octen-1-ol (D), nonanal (E), linalool (F), $n$-(3-methylbutyl)acetamide (G), bornyl acetate (H), 2,4-decadienal (I), geranyl acetate (J) by examination of another independent set of samples. 
predicted values obtained from prediction models were compared with the reference values determined by GC-FID analysis. The predicted values versus reference values are shown in Fig. 6.

The root mean square error of prediction (RMSEP) represents the accuracy of a prediction model and indicates the average difference between predicted values and reference values. ${ }^{47}$ A satisfied model should have a high value of $R^{2}$ and a low value of RMSEP. As shown in Fig. 6, it was observed that the reference data points for geranyl acetone were closer to the regression line, which indicated that the reference values and predicted values for geranyl acetone were in good agreement. The correlation coefficient $(R=0.97)$, regression coefficient $\left(R^{2}\right.$ $=0.93$ ) and RMSEP (1.10) for geranyl acetone were quite satisfactory for the validation of the model. Furthermore, the model validation for 1-octene, 2-octen-1-ol, 2-pentylfuran, linalool, bornyl acetate and nonanal showed a high correlation coefficient $(R>0.92)$ and regression coefficient $\left(R^{2}>0.84\right)$ (Fig. 6). These results indicated the established prediction models were suitable for performing prediction and provided predictability levels of $82 \%$ for 1 -octene, $88 \%$ for 2 -pentylfuran, $88 \%$ for 2 octen-1-ol, $87 \%$ for linalool, $84 \%$ for nonanal, $87 \%$ for bornyl acetone, $84 \%$ for 2,4 -decadienal and $92 \%$ for geranyl acetate. For $n$-(3-methylbutyl)acetamide, the correlation coefficient $(R=$ $0.89)$ and regression coefficient $\left(R^{2}=0.78\right)$ were considered to be satisfactory, however, its RMSEP (about 22.87) was relatively poor. This might be due to the formation of $n$-(3-methylbutyl) acetamide related to the Maillard reaction during the drying process, ${ }^{45}$ which was impossible to be totally controlled, resulting in the nonlinear relationship under different SFE parameters.

\section{Conclusions}

The influence of SFE extraction conditions on the extraction yield and the content of mushroom alcohols was evaluated. The results revealed that higher pressure (85 bar), lower temperature $\left(40{ }^{\circ} \mathrm{C}\right)$ and higher $\mathrm{SC}-\mathrm{CO}_{2}$ volume $(6 \mathrm{~g}$ samples $/ 50 \mathrm{~mL} \mathrm{SC}$ $\mathrm{CO}_{2}$ ) were the best conditions for the satisfactory yield and sufficient high content of mushroom alcohols in the extracts.

The relationship between supercritical fluid parameters and the extraction yield of typical mushroom volatiles was studied using PLSR to point out the significant factors correlated to the yield of each aroma compound. Prediction models were established to estimate the yield of volatiles under varying parameters of SFE, and satisfactory results were obtained for 1-octene, 1-octen-3-ol, 2-pentylfuran, 2-octen-1-ol, linalool, nonanal, bornyl acetate, 2,4-decadiena, $n$-(3-methylbutyl)acetamide, and geranyl acetone. Furthermore, another independent data set was applied to validate the prediction models. A good prediction ability was obtained for most of the volatile compounds including 1-octene, 1-octen-3-ol, 2-pentylfuran, 2-octen-1-ol, linalool, nonanal, bornyl acetate, 2,4-decadienal, and geranyl acetone. The predictable equation could be a promising tool to predict the yield of extracted volatile compounds from mushrooms. In addition, the developed models can be applied to facilitate the selectivity of supercritical fluid extraction of target compounds from the wild Finnish mushroom Craterellus tubaeformis by adjusting favourable parameters. This is the first study focusing on the supercritical fluid extraction of volatile compounds from the wild Finnish mushroom species Craterellus tubaeformis.

\section{Conflicts of interest}

The authors declare no competing financial interest and there are no conflicts to declare.

\section{Acknowledgements}

The laboratory work was carried out at the Food Chemistry and Food Development Unit at the University of Turku, Finland. The research was part of the project "Innovative Technologies and Concepts for Business Growth Based on Finnish Mushrooms" funded by TEKES - the Finnish Funding Agency for Innovation and food companies from Finland and Germany (Dnro 3135/31/ 2015). The Strategic Funding Programme of University of Turku "Finnish-Chinese Collaboration in Food Quality and Safety" and scholarship grant of Jiangnan University (China) are also warmly acknowledged for financing support. The authors sincerely thank Heikki Kallio for support in the supercritical fluid extraction and Heikki Aisala for assistance in GC-MS and GC-FID analysis.

\section{References}

1 http:/www.businesswire.com/news/home/20150121005309/ en/Research-Markets-Global-Mushroom-Market-TrendsForecasts, accessed 24.08.2017.

2 S. Chatterjee, M. K. Sarma, U. Deb, G. Steinhauser, C. Walther and D. K. Gupta, Environ. Sci. Pollut. Res., 2017, 24, 19480-19493.

3 P. Mattila, P. Salo-Väänänen, K. Könkö, H. Aro and T. Jalava, Food Chem., 2002, 50, 6419-6422.

4 T. C. Sarma, I. Sarma and B. N. Patiri, Bioscan, 2010, 3, 613625.

5 P. H. Mattila, V. I. Piironen, E. J. Uusi-Rauva and P. E. Koivistoinen, Food Chem., 1994, 42, 2449-2453.

6 T. A. Outila, P. H. Mattila, V. I. Piironen and C. J. LambergAllardt, Am. J. Clin. Nutr., 1999, 69, 95-98.

7 M. E. Valverde, T. Hernández-Pérez and O. Paredes-López, Int. J. Microbiol., 2015, 2015, 1-14.

8 J. A. Maga, J. Agric. Food Chem., 1981, 29, 1-4.

9 A. Usami, S. Nakaya, H. Nakahashi and M. Miyazawa, J. Oleo Sci., 2014, 63, 1323-1332.

10 R. Tressl, D. Bahri and K. H. Engel, Food Chem., 1982, 30, 8993.

11 J. S. Dickschat, Nat. Prod. Rep., 2017, 34, 310-328.

12 P. Kalač, Food Chem., 2009, 113, 9-16.

13 M. V. Chandravadana, G. Vekateshwarlu, C. S. B. Babu, T. K. Roy, K. S. Shivashankara, M. Pandey, R. P. Tewari and Y. Selvaraj, Flavour Fragrance J., 2005, 20, 715-717.

14 A. Usami, R. Motooka, H. Nakahashi, Y. Okuno and M. Miyazawa, J. Oleo Sci., 2014, 63, 731-739. 
15 S. Nosaka and M. Miyazawa, J. Oleo Sci., 2014, 63, 577-583. 16 O. Taofiq, A. M. González-Paramás, A. Martins, M. F. Barreiro and I. C. F. R. Ferreira, Ind. Crops Prod., 2016, 90, 38-48.

17 H. E. Nursten and M. L. Woolfe, J. Sci. Food Agric., 1972, 23, 803-822.

18 H. Nakahashi, Y. Nishino, H. Nakagawa, N. Hara, A. Usami and M. Miyazawa, J. Oleo Sci., 2015, 64, 1235-1242.

19 H. Krüger, Planta Med., 2010, 76, 843-846.

20 A. Usami, T. Ono, Y. Kashima, H. Nakahashi, S. Marumoto, S. Nosaka, S. Watanabe and M. Miyazawa, J. Oleo Sci., 2014, 63, 83-92.

21 Q. Lang and C. M. Wai, Talanta, 2001, 53, 771-782.

22 S. M. Pourmortazavi and S. S. Hajimirsadeghi, J. Chromatogr. A, 2007, 1163, 2-24.

23 M. M. R. de Melo, A. J. D. Silvestre and C. M. Silva, J. Supercrit. Fluids, 2014, 92, 115-176.

24 R. P. F. F. da Silva, T. A. P. Rocha-Santos and A. C. Duarte, Trends Anal. Chem., 2016, 76, 40-51.

25 E. Uquiche, N. Cirano and S. Millao, Ind. Crops Prod., 2015, 77, 307-314.

26 Z. Zeković, B. Pavlić, A. Cvetanović and S. Đurović, Ind. Crops Prod., 2016, 94, 353-362.

27 J. Shi, M. Khatri, S. J. Xue, G. S. Mittal, Y. Ma and D. Li, Sep. Purif. Technol., 2009, 66, 322-328.

28 M. Miyazawa, Y. Kawauchi and N. Matsuda, Flavour Fragrance J., 2010, 25, 197-201.

29 P. G. de Pinho, B. Ribeiro, R. F. Goncalves, P. Baptista, P. Valentao, R. M. Seabra and P. B. Andrade, J. Agric. Food Chem., 2008, 56, 1704-1712.

30 C. S. G. Kitzberger, R. H. Lomonaco, E. M. Z. Michielin, L. Danielski, J. Correia and S. R. S. Ferreira, J. Food Eng., 2009, 90, 35-43.

31 C. S. G. Kitzberger, A. Smânia, R. C. Pedrosa and S. R. S. Ferreira, J. Food Eng., 2007, 80, 631-638.
32 D. Morales, A. Gil-Ramirez, F. R. Smiderle, A. J. Piris, A. RuizRodriguez and C. Soler-Rivas, Innovative Food Sci. Emerging Technol., 2017, 41, 330-336.

33 S. Lin and X. Zhang, Anal. Methods, 2016, 8, 1609-1617.

34 S. Song, X. Zhang, K. Hayat, M. Huang, P. Liu, E. Karangwa, F. Gu, C. Jia, S. Xia, Z. Xiao and Y. Niu, J. Chromatogr. A, 2010, 1217, 7788-7799.

35 J. H. Cheng and D. W. Sun, J. Food Eng., 2017, 9, 36-49.

36 Peace software, http:/www.peacesoftware.de/einigewerte/ co2_e.html, accessed 24.08.2017.

37 M. Bocevska and H. Sovová, J. Supercrit. Fluids, 2007, 40, 360-367.

38 N. M. Shrigod, N. R. Swami Hulle and R. V. Prasad, J. Food Process Eng., 2017, 40, 1-9.

39 D. Villanueva-Bermejo, F. Zahran, M. R. García-Risco, G. Reglero and T. Fornari, J. Supercrit. Fluids, 2017, 119, 283-288.

40 K. Przygoda and G. Wejnerowska, Ind. Crops Prod., 2015, 63, 41-47.

41 R. Salea, B. Veriansyah and R. R. Tjandrawinata, J. Supercrit. Fluids, 2017, 120, 285-294.

42 K. Lisichkov, S. Kuvendziev, Z. Zeković and M. Marinkovski, Sep. Purif. Technol., 2014, 138, 191-197.

43 M. Hamburger, D. Baumann and S. Adler, Phytochem. Anal., 2004, 15, 46-54.

44 I. H. Cho, S. M. Lee, S. Y. Kim, H. K. Choi, K. O. Kim and Y. S. Kim, J. Agric. Food Chem., 2007, 55, 2323-2328.

45 M. Miyazawa, Y. Dejima, T. Takahashi, N. Matsuda and R. Ishikawa, J. Essent. Oil Res., 2011, 23, 58-63.

46 D. K. Dandge, J. P. Heller and K. V. Wilson, Industrial and Engineering Chemistry Product Research and Development, 1985, vol. 24, pp. 162-166.

47 The Unscrambler tutorials, 2006, pp. 14-16. 